\title{
A NOTE ON SUB-BUNDLES OF VECTOR BUNDLES
}

\author{
WILLIAM CRAWLEY-BOEVEY \\ Department of Pure Mathematics, University of Leeds, Leeds LS29JT, United Kingdom
}

and BERNT TORE JENSEN

Département de mathématiques, Faculté des Sciences, Université de Sherbrooke, 2500 boul. de l'Université, Sherbrooke (Quebec) J1K2R1, Canada

e-mail: Bernt.Tore.Jensen@USherbrooke.ca

(Received 3 February, 2006; accepted 27 April, 2006)

\begin{abstract}
It is easy to imagine that a subvariety of a vector bundle, whose intersection with every fibre is a vector subspace of constant dimension, must necessarily be a sub-bundle. We give two examples to show that this is not true, and several situations in which the implication does hold. For example it is true if the base is normal and the field has characteristic zero. A convenient test is whether or not the intersections with the fibres are reduced as schemes.
\end{abstract}

2000 Mathematics Subject Classification. 14F05.

Introduction. Let $X$ be a connected algebraic variety over an algebraically closed field $k$. Recall that a vector bundle of rank $n$ on $X$ is a variety $E$ equipped with a morphism $\pi: E \rightarrow X$ and the structure of an $n$-dimensional vector space on each fibre $E_{x}=\pi^{-1}(x)$, satisfying the local triviality condition that each $x \in X$ has an open neighbourhood $U$ such that there is an isomorphism $\phi: \pi^{-1}(U) \rightarrow U \times k^{n}$ making the diagram

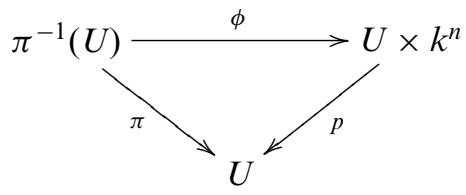

commute, where $p$ is the projection, and with $\phi$ inducing linear maps on the fibres over $U$.

A sub-bundle of $E$ is a closed subvariety $F$ of $E$ whose fibres $F_{x}=F \cap E_{x}$ are vector subspaces of the $E_{x}$ for all $x \in X$, and such that $F$, equipped with the map $\left.\pi\right|_{F}: F \rightarrow X$ and the induced vector space structures on its fibres, is locally trivial, so a vector bundle.

It is easy to imagine that

(*) $F$ is a closed subvariety of $E$ and the $F_{x}$ are vector subspaces of the $E_{x}$ of constant dimension for all $x \in X$,

implies that $F$ is a sub-bundle of $E$. We give two examples showing that this implication is not true in general, and several situations in which it does hold. For example it is 
true if $X$ is normal and $k$ has characteristic zero. A convenient test is whether or not the intersections with the fibres are reduced as schemes.

1. Two general situations. The first situation is well known.

PROPOSITION 1. If F satisfies (*), then $F$ is a sub-bundle if and only if $F$ is the kernel of a vector bundle homomorphism $\theta: E \rightarrow E^{\prime}$.

If $F$ is a sub-bundle, one can take $E^{\prime}$ to be the quotient bundle $E / F$. For the converse see for example [3, Proposition 1.7.2].

Proposition 2. If $F$ satisfies $\left(^{*}\right)$, then $F$ is a sub-bundle if and only if for all $x \in X$ the natural scheme structure on $F_{x}$, as an intersection of varieties $F \cap E_{x}$, or equivalently as a fibre $\left(\left.\pi\right|_{F}\right)^{-1}(x)$, is reduced.

Proof. Clearly if $F$ is sub-bundle then local triviality implies that the $F_{x}$ are reduced. For the converse, since the statement is local on the base, we may assume that $X$ is an affine variety and $E=X \times k^{n}$ is trivial. Let $y=\left(y_{1}, \ldots, y_{n}\right)$ be coordinates for $k^{n}$ and let $f_{1}, \ldots, f_{s} \in k[X]\left[y_{1}, \ldots, y_{n}\right]$ be a generating set for the ideal defining $F$.

For fixed $x$, we identify $E_{x}$ with $k^{n}$, and then $f_{1}, \ldots, f_{s}$ define $F_{x}$ as a closed subscheme of $k^{n}$. The tangent space of $F_{x}$ at 0 is

$$
T_{0} F_{x}=\left\{y \in k^{n}: J(x) y=0\right\},
$$

where $J(x)$ is the $s \times n$ matrix with

$$
J(x)_{i j}=\left.\frac{\partial f_{i}(x, y)}{\partial y_{j}}\right|_{y=0} .
$$

We show that $F_{x}=T_{0} F_{x}$. If $y \in F_{x}$, then so is $t y$ for all $t \in k$, so $f_{i}(x, t y)=0$ for all $i$ and $t$. Expanding in powers of $t$, and taking the linear terms, we deduce that $J(x) y=0$, so $F_{x} \subseteq T_{0} F_{x}$. Now this is an equality since the scheme structure of $F_{x}$ is assumed to be reduced, and the variety structure is that of a vector space, so $F_{x}$ is smooth, and hence $\operatorname{dim} T_{0} F_{x}=\operatorname{dim} F_{x}$.

Now Proposition 1 applies, since $F$ is the kernel of the vector bundle homomorphism $\theta: X \times k^{n} \rightarrow X \times k^{s}, \theta(x, y)=(x, J(x) y)$.

We observe that in the formulation of $\left(^{*}\right)$ we could have used the weaker condition that $F$ is locally closed in $E$.

PROPOSITION 3. If $F$ is a locally closed subvariety of $E$ and the $F_{x}$ are vector subspaces of the $E_{x}$ of constant dimension for all $x \in X$, then $F$ is closed in $E$.

Proof. Scalar multiplication on the fibres defines a map $m: k \times E \rightarrow E$, and since the fibres of $F$ are subspaces, we have $m(k \times F)=F$. It follows that $m(k \times \bar{F})=\bar{F}$, so that $\bar{F} \cap E_{x}$ is closed under scalar multiplication in the fibre $E_{x}$, and hence must be connected. Now $F_{x}$ is an open subset of $\bar{F} \cap E_{x}$ since $F$ is locally closed in $E$, and a closed subset since $F_{x}$ is a vector subspace of $E_{x}$. Thus by connectedness, $F_{x}=\bar{F} \cap E_{x}$, so $F=\bar{F}$.

2. Normal base in characteristic zero. We need the following form of Zariski's Main Theorem. For a proof see for example [2, Section III.9]. 
LEMMA 1. If $X$ is normal and $f: X^{\prime} \longrightarrow X$ is a birational morphism with finite fibres, then $f$ is an isomorphism of $X^{\prime}$ with an open subset $U \subseteq X$.

LEMMA 2. If $F$ satisfies $(*)$ and $X$ is irreducible, then so is $F$.

Proof. Let $d$ be the dimension of the $F_{x}$, and let $Z_{1}, \ldots, Z_{m}$ be the irreducible components of $F$. Applying the theorem on upper semicontinuity of fibre dimensions to $\left.\pi\right|_{Z_{i}}$, see [2, Section I.8, Corollary 3], we see that the set

$$
G_{i}=\left\{z \in Z_{i} \mid \operatorname{dim}_{z}\left(Z_{i} \cap \pi^{-1}(\pi(z))\right) \geq d\right\}
$$

is closed in $Z_{i}$, where $\operatorname{dim}_{z} Y$ denotes the maximum of the dimensions of the irreducible components of $Y$ which contain $z$.

Since $m\left(k \times Z_{i}\right)$ contains $Z_{i}$, and its closure is an irreducible closed subset of $F$, we must have $m\left(k \times Z_{i}\right)=Z_{i}$. Thus the intersection of $Z_{i}$ with any fibre $E_{x}$ is closed under scalar multiplication, and hence any irreducible component of this intersection contains the zero element. Thus if we define

$$
X_{i}=\left\{x \in X \mid \operatorname{dim}\left(Z_{i} \cap \pi^{-1}(x)\right) \geq d\right\}
$$

and $s: X \rightarrow E$ denotes the zero section, then

$$
X_{i}=\left\{x \in X \mid \operatorname{dim}_{s(x)}\left(Z_{i} \cap \pi^{-1}(x)\right) \geq d\right\}=s^{-1}\left(G_{i}\right),
$$

so that $X_{i}$ is closed in $X$.

Now if $x \in X$, then $F_{x}=\bigcup_{i=1}^{m}\left(Z_{i} \cap \pi^{-1}(x)\right)$, so $\operatorname{dim} Z_{i} \cap \pi^{-1}(x) \leq d$. Moreover, since $F_{x}$ is irreducible, equality must hold for some $i$, and then $F_{x}=Z_{i} \cap \pi^{-1}(x)$. It follows that $X=\bigcup_{i=1}^{m} X_{i}$, so since $X$ is irreducible, $X=X_{i}$ for some $i$, and then $F=Z_{i}$, so it is irreducible.

Proposition 4. If $F$ satisfies $(*)$, then $F$ is a sub-bundle if the field $k$ has characteristic zero and $X$ is normal.

Proof. We have a standing assumption that $X$ is connected, so since $X$ is normal it is irreducible. The statement is local on the base, so by passing to a neighbourhood of $x_{0} \in X$, we may assume that $E=X \times k^{n}$ is trivial. For each $x \in X$ identify $F_{x}$ as a vector subspace of $k^{n}$. Fix a complement $C$ of $F_{x_{0}}$ in $k^{n}$.

Applying the upper semicontinuity theorem to the restriction of $\pi$ to $F \cap(X \times C)$ we see that $F_{x} \cap C=\{0\}$ for all $x$ in a neighbourhood of $x_{0}$. Then, by shrinking $X$ again, we may assume that $F_{x} \oplus C=k^{n}$ for all $x \in X$.

Let $\phi: F \rightarrow X \times F_{x_{0}}$ be the restriction to $F$ of the map $X \times k^{n} \rightarrow X \times F_{x_{0}}$ given by the projection perpendicular to $C$. Clearly $\phi$ is a bijective morphism of varieties. Now $F$ is irreducible by Lemma 2, so $\phi$ is a bijective morphism between irreducible varieties over a field of characteristic zero, which shows that $\phi$ is birational by [1, Proposition 7.16]. Then $\phi$ is an isomorphism by Zariski's Main Theorem. This shows that $F$ is locally trivial, and hence a sub-bundle.

\section{Examples.}

EXAMPLE 1. Let $X$ be the cuspidal curve $y^{2}=x^{3}$, and let

$$
F=\left\{(x, y, z, w) \mid y^{2}=x^{3}, y z=x w, w^{2}=x z^{2}, x^{2} z=y w\right\} \subseteq X \times k^{2} .
$$


We need to know that these equations define $F$ with its reduced scheme structure, so that they induce the natural scheme structure on the fibres of $F$. For this it suffices to show that the ideal corresponding to these equations is the kernel of the homomorphism $k[x, y, z, w] \rightarrow k[t, z]$ sending $x, y, z, w$ to $t^{2}, t^{3}, z, t z$ respectively. We leave the details as an exercise to the reader.

Now the fibre of $F$ over a point $(x, y)=\left(t^{2}, t^{3}\right)$ with $t \neq 0$ is the line $w=t z$ in $k^{2}$, while the fibre over the point $(0,0)$ is the subscheme of $k^{2}$ defined by the equation $w^{2}=0$. As a subset this is the line $w=0$, so every fibre of $F$ is a 1-dimensional subspace of $k^{2}$. Since the fibre of $F$ over $(0,0)$ is not reduced, $F$ cannot be a sub-bundle of $X \times k^{2}$.

EXAMPLE 2. Let $k$ be a field of characteristic $p>0$, and let $X=k$, the affine line. Let

$$
F=\left\{(x, y, z): y^{p}=x z^{p}\right\} \subseteq k \times k^{2} .
$$

Since $y^{p}-x z^{p}$ is an irreducible polynomial, the equation defines $F$ with its reduced scheme structure.

The fibre over any point $x=\lambda \in k$ is the line $y=\lambda^{1 / p} z$, but the scheme structure is given by the equation $y^{p}=\lambda z^{p}$, or equivalently $\left(y-\lambda^{1 / p} z\right)^{p}=0$, so it is not reduced. Thus $F$ is not a sub-bundle.

\section{REFERENCES}

1. Joe Harris, Algebraic geometry. A first course. Graduate Texts in Mathematics $\mathbf{1 3 3}$ (Springer-Verlag, 1995).

2. David Mumford, The red book of varieties and schemes. Second, expanded edition. Lecture Notes in Mathematics No. 1358 (Springer-Verlag, 1999).

3. Joseph Le Potier, Lectures on vector bundles. Cambridge Studies in Advanced Mathematics 54 (Cambridge University Press, 1997). 